\title{
Exploring the origin of magnetic fields in massive stars: a survey of O-type stars in clusters and in the field ${ }^{\star}$
}

\author{
S. Hubrig ${ }^{1}$, M. Schöller ${ }^{2}$, N. V. Kharchenko ${ }^{1,3}$, N. Langer ${ }^{4}$, W. J. de Wit ${ }^{5}$, I. Ilyin ${ }^{1}$, A. F. Kholtygin ${ }^{6}$, A. E. Piskunov ${ }^{1,7}$, \\ N. Przybilla ${ }^{8}$, and the MAGORI collaboration
}

\author{
1 Astrophysikalisches Institut Potsdam, An der Sternwarte 16, 14482 Potsdam, Germany \\ e-mail: shubrig@aip.de \\ 2 European Southern Observatory, Karl-Schwarzschild-Str. 2, 85748 Garching, Germany \\ 3 Main Astronomical Observatory, 27 Academica Zabolotnogo Str., 03680 Kiev, Ukraine \\ 4 Argelander-Institut für Astronomie, Universität Bonn, Auf dem Hügel 71, 53121 Bonn, Germany \\ 5 European Southern Observatory, Alonso de Cordova 3107, Santiago, Chile \\ 6 Astronomical Institute, Saint-Petersburg State University, Saint-Petersburg, Russia \\ 7 Institute of Astronomy of the Russian Acad. Sci., 48 Pyatnitskaya Str., 109017 Moscow, Russia \\ ${ }^{8}$ Dr.-Karl-Remeis-Sternwarte Bamberg \& ECAP, Universität Erlangen-Nürnberg, Sternwartstr. 7, 96049 Bamberg, Germany
}

Received 17 December 2010 / Accepted 7 February 2011

\begin{abstract}
Context. Although the effects of magnetic fields in massive stars have been found to be substantial by recent models and observations, the magnetic fields of only a small number of massive O-type stars have so far been investigated. Additional observations are of the utmost importance to constraining the conditions that are conducive to magnetic fields and to determine the first trends about their occurrence rate and field strength distribution.

Aims. To investigate statistically whether magnetic fields in massive stars are ubiquitous or appear only in stars with a specific spectral classification, certain ages, or in a special environment, we acquired 41 new spectropolarimetric observations for 36 stars. Among the observed sample, roughly half of the stars are probable members of clusters at different ages, whereas the remaining stars are field stars not known to belong to any cluster or association.

Methods. Spectropolarimetric observations were obtained during three different nights using the low-resolution spectropolarimetric mode of FORS 2 (FOcal Reducer low dispersion Spectrograph) mounted on the 8-m Antu telescope of the VLT. To assess the membership in open clusters and associations, we used astrometric catalogues with the highest quality kinematic and photometric data currently available.

Results. A field at a significance level of $3 \sigma$ was detected in ten O-type stars. The strongest longitudinal magnetic fields were measured in two Of?p stars: $\left\langle B_{z}\right\rangle=-381 \pm 122 \mathrm{G}$ for CPD-282561 and $\left\langle B_{z}\right\rangle=-297 \pm 62 \mathrm{G}$ for HD 148937, the latter of which had previously been detected by ourselves as magnetic. The observations of HD 148937 obtained on three different nights indicate that the magnetic field is slightly variable. Our new measurements support our previous conclusion that large-scale organized magnetic fields with polar field strengths in excess of $1 \mathrm{kG}$ are not widespread among O-type stars. Among the stars with a detected magnetic field, only one star, HD 156154, belongs to an open cluster at high membership probability. According to previous kinematic studies, four magnetic O-type stars in the sample are well-known candidate runaway stars.
\end{abstract}

Key words. polarization - stars: magnetic field - stars: kinematics and dynamics - open clusters and associations: general stars: early-type

\section{Introduction}

Magnetic fields play an important role in astrophysical phenomena of the universe on various scales. For galaxies, dynamo models associated with various MHD instabilities occurring in the interstellar medium (ISM) are used to explain the formation of the galactic structure (e.g., Gomez \& Cox 2004; Bonanno \& Urpin 2008). Magnetic fields play a role in the evolution of interstellar molecular clouds and the star formation process, where the cloud collapse is probably taking place along the magnetic field lines (e.g. Alves et al. 2008). They are also present at all stages of stellar evolution, from young T Tauri stars and Ap/Bp stars to the end products: white dwarfs, neutron stars, and magnetars. On the other hand, the role of magnetic fields in massive

* Based on observations obtained at the European Southern Observatory, Paranal, Chile (ESO programme 085.D-0667(A)).
O-type stars and Wolf-Rayet (WR) stars remains unknown. No definitive magnetic field has ever been detected in WR stars and presently only less than a dozen $\mathrm{O}$ stars have published magnetic fields. In addition, the theories about the origin of magnetic fields in O-type stars remain poorly developed, mostly because the distribution of magnetic field strengths in massive stars from the ZAMS to more evolved stages has not yet been studied.

In our study, we focus on magnetic fields of massive stars observed in different environments: in open clusters at different ages and in the field. The results of our recent kinematic analysis of known magnetic O-type stars using the best available astrometric, spectroscopic, and photometric data indicates that a magnetic field is more frequently detected in candidate runaway stars than in stars belonging to clusters or associations (Hubrig et al. 2011a). As the sample of stars with magnetic field detections is still very small, a study of a larger sample is urgently 
needed to confirm the detected trend by performing dedicated magnetic field surveys of $\mathrm{O}$ stars in clusters/associations and the field. We were granted four nights in 2010 May with FORS 2 at the VLT to survey magnetic fields in massive stars, but because of poor weather conditions, only half of the granted time could be used for observations. Notwithstanding, the results obtained allow us to constrain preliminarily the conditions conducive to the presence of magnetic fields and derive the first trends for their occurrence rate and field strength distribution. This information is critical for answering the principal question of the possible origin of magnetic fields in massive stars.

In the following, we present 41 new measurements of magnetic fields in 36 massive stars using FORS 2 at the VLT in spectropolarimetric mode. Our observations and the obtained results are described in Sect. 2, and their discussion is presented in Sect. 3 .

\section{Observations and results}

Spectropolarimetric observations were carried out in 2010 May 20-23 in visitor mode at the European Southern Observatory with FORS 2 mounted on the 8-m Antu telescope of the VLT. This multi-mode instrument is equipped with polarization analyzing optics, comprising super-achromatic half-wave and quarter-wave phase retarder plates, and a Wollaston prism with a beam divergence of $22^{\prime \prime}$ in standard resolution mode ${ }^{1}$. Polarimetric spectra were obtained with the GRISM 600B and the narrowest slit width of 0.4 to achieve a spectral resolving power of $R \sim 2000$. The use of the mosaic detector made of blue optimized E2V chips and a pixel size of $15 \mu \mathrm{m}$ allowed us to cover a large spectral range, from 3250 to $6215 \AA$, which includes all hydrogen Balmer lines from $\mathrm{H} \beta$ to the Balmer jump. The spectral types and the visual magnitudes of the studied stars are listed in Table 1.

A detailed description of the assessment of the longitudinal magnetic-field measurements using FORS 2 was presented in our previous papers (e.g., Hubrig et al. 2004a,b, and references therein). The mean longitudinal magnetic field, $\left\langle B_{z}\right\rangle$, was derived using

$\frac{V}{I}=-\frac{g_{\mathrm{eff}} e \lambda^{2}}{4 \pi m_{\mathrm{e}} c^{2}} \frac{1}{I} \frac{\mathrm{d} I}{\mathrm{~d} \lambda}\left\langle B_{z}\right\rangle$,

where $V$ is the Stokes parameter that measures the circular polarisation, $I$ is the intensity in the unpolarised spectrum, $g_{\mathrm{eff}}$ is the effective Landé factor, $e$ is the electron charge, $\lambda$ is the wavelength, $m_{\mathrm{e}}$ the electron mass, $c$ the speed of light, $\mathrm{d} I / \mathrm{d} \lambda$ is the derivative of Stokes $I$, and $\left\langle B_{z}\right\rangle$ is the mean longitudinal magnetic field.

Longitudinal magnetic fields were measured in two ways: using only the absorption hydrogen Balmer lines or the entire spectrum including all available absorption lines. The lines that show evidence of emission were not used to determine the magnetic field strength. The feasibility of longitudinal magnetic field measurements in massive stars using low-resolution spectropolarimetric observations has been demonstrated by previous studies of O and B-type stars (e.g., Hubrig et al. 2006, 2008, 2009a, 2011b). To verify that the instrument was functioning properly, we observed the magnetic Ap star HD 187474, which has a well-studied longitudinal magnetic field, during the night of May 23 at rotation phase 0.66 . HD 187474 has a rotation period of $6.4 \mathrm{yr}$ and a longitudinal magnetic field ranging roughly

\footnotetext{
1 The spectropolarimetric capabilities of FORS 1 were moved to FORS 2 in 2009.
}

Table 1. List of O-type stars observed with FORS 2.

\begin{tabular}{|c|c|c|c|}
\hline Name & $\begin{array}{c}\text { Other } \\
\text { Identifier }\end{array}$ & $\bar{V}$ & Spectral Type \\
\hline CPD-282561 & SAO 174826 & 10.09 & O6.5 fp \\
\hline CPD-47 2963 & SAO 220701 & 8.45 & O4 III (f) \\
\hline CPD-58 2620 & CD-583529 & 9.27 & O6.5 V ((f)) \\
\hline HDE 303311 & CPD-58 2652 & 8.98 & $\mathrm{O} 5 \mathrm{~V}$ \\
\hline HD 93129B & CPD-58 2618B & 7.16 & O3.5 V $((f+))$ \\
\hline HD 93204 & CPD-59 2584 & 8.48 & O5 V ((f)) \\
\hline HDE 303308 & CPD-59 2623 & 8.14 & O4 V ((f+)) \\
\hline HD 93403 & CPD-58 2680 & 7.30 & O5 III (f) var sec \\
\hline HD 93843 & CPD-592732 & 7.33 & O5 III(f) var \\
\hline HD 105056 & GS Mus & 7.34 & ON9.7 Ia e \\
\hline HD 115455 & CPD-613575 & 7.97 & O7.5 III ((f)) \\
\hline HD 120521 & CPD-57 6339 & 8.56 & O8 Ib (f) \\
\hline HD 123590 & CPD-61 4382 & 7.62 & O7/8 \\
\hline HD 125206 & CPD-605298 & 7.94 & O9.5 IV: (n) \\
\hline HD 125241 & CPD-60 5300 & 8.31 & O8.5 Ib (f) \\
\hline HD 130298 & CPD-55 6191 & 9.29 & O6.5 III (n)(f) \\
\hline HD 148937 & CPD-47 7765 & 6.77 & O6.5 f?p \\
\hline HD 328856 & CPD-46 8218 & 8.50 & O9.5III* \\
\hline HD 152233 & CPD-41 7718 & 6.59 & O6 III: (f)p \\
\hline HD 152247 & CPD-41 7732 & 7.20 & O9.5 II-III \\
\hline HD 152249 & HR 6263 & 6.47 & OC9.5 Iab \\
\hline HD 153426 & CPD-38 6624 & 7.49 & O9 II-III \\
\hline HD 153919 & V884 Sco & 6.53 & O6.5 Ia f+ \\
\hline HD 154368 & V1074 Sco & 6.18 & O9.5 Iab \\
\hline HD 154643 & CPD-34 6733 & 7.15 & O9.5 III \\
\hline HD 154811 & CPD-468416 & 6.93 & OC9.7 Iab \\
\hline HD 156154 & CPD-35 6916 & 8.04 & O8 Iab (f) \\
\hline HD 156212 & CPD-27 5605 & 7.95 & O9.7 Iab \\
\hline HD 165319 & BD-144880 & 7.93 & O9.5Iab* \\
\hline HD 315033 & CPD-24 6163 & 8.90 & B3* \\
\hline HD 168075 & BD-13 4925 & 8.73 & O6 V ((f)) \\
\hline HD 168112 & BD-124988 & 8.55 & O5 III (f) \\
\hline HD 169582 & BD-094729 & 8.70 & O6 If \\
\hline HD 171589 & BD-145131 & 8.21 & O7 II (f) \\
\hline HD 175754 & BD-195242 & 7.02 & O8 II ((f)) \\
\hline HD 187474 & V3961 Sgr & 5.32 & $\mathrm{Ap}^{*}$ \\
\hline
\end{tabular}

Notes. Spectral classifications are listed according to the Galactic O Star Catalogue (Maíz-Apellániz et al. 2004). * Indicates Spectral Types taken from SIMBAD.

from $-2 \mathrm{kG}$ to $2 \mathrm{kG}$. The measured value of the magnetic field, $\left\langle B_{z}\right\rangle_{\mathrm{all}}=-1249 \pm 47 \mathrm{G}$, fits very well to the observations at the same phase presented by Landstreet \& Mathys (2000).

Although we were granted four nights for our survey, owing to unfavorable weather conditions (snow and high humidity) only four stars could be observed during the first night, 14 stars during the second night, none during the third night, and 23 during the last night. Most of the targets were observed only once. The exceptions were the stars HD 328856, HD 153426, and HD 168112, which we were able to observe twice. HD 148937 was observed three times to assess the magnetic field variability over the rotation cycle. Apart from this star, which has a rotation period of seven days (Nazé et al. 2008), no exact rotation periods are known for the other stars in our sample.

The results of our magnetic field measurements are presented in Table 2. In the first two columns, we provide the star names and the modified Julian dates at the middle of the exposures. In Cols. 3 and 4 , we present the longitudinal magnetic field $\left\langle B_{z}\right\rangle_{\text {all }}$ using the whole spectrum, and the longitudinal magnetic field $\left\langle B_{z}\right\rangle_{\text {hyd }}$ using only the hydrogen lines. All quoted errors are $1 \sigma$ uncertainties. In Col. 5, we identify new detections by ND and 
Table 2. Longitudinal magnetic fields measured with FORS 2 in the studied sample.

\begin{tabular}{|c|c|c|c|c|}
\hline Name & $\overline{\mathrm{MJD}}$ & $\begin{array}{c}\left\langle B_{z}\right\rangle_{\text {all }} \\
{[\mathrm{G}]}\end{array}$ & $\begin{array}{c}\left\langle B_{z}\right\rangle_{\text {hydr }} \\
{[\mathrm{G}]}\end{array}$ & Comment \\
\hline CPD-282561 & 55338.969 & $-381 \pm 122$ & $-534 \pm 167$ & ND \\
\hline CPD-47 2963 & 55337.094 & $-190 \pm 62$ & $-154 \pm 96$ & ND \\
\hline CPD-58 2620 & 55339.020 & $-53 \pm 71$ & $-66 \pm 88$ & \\
\hline HDE 303311 & 55337.131 & $-56 \pm 40$ & $-19 \pm 61$ & \\
\hline HD 93129B & 55337.179 & $-49 \pm 44$ & $-79 \pm 78$ & \\
\hline HD 93204 & 55339.053 & $22 \pm 46$ & $16 \pm 66$ & \\
\hline HDE 303308 & 55339.078 & $122 \pm 54$ & $137 \pm 96$ & \\
\hline HD 93403 & 55339.116 & $39 \pm 41$ & $-15 \pm 88$ & \\
\hline HD 93843 & 55339.099 & $-157 \pm 42$ & $-173 \pm 56$ & ND \\
\hline HD 105056 & 55337.208 & $-93 \pm 48$ & $-156 \pm 63$ & \\
\hline HD 115455 & 55337.230 & $4 \pm 47$ & $13 \pm 64$ & \\
\hline HD 120521 & 55337.257 & $25 \pm 44$ & $-3 \pm 62$ & \\
\hline HD 123590 & 55339.133 & $19 \pm 42$ & $56 \pm 70$ & \\
\hline HD 125206 & 55337.307 & $12 \pm 64$ & $8 \pm 91$ & \\
\hline HD 125241 & 55339.188 & $24 \pm 39$ & $16 \pm 72$ & \\
\hline HD 130298 & 55339.159 & $113 \pm 38$ & $193 \pm 62$ & ND \\
\hline \multirow[t]{3}{*}{ HD 148937} & 55336.307 & $-297 \pm 62$ & $-293 \pm 85$ & $\mathrm{CD}$ \\
\hline & 55337.285 & $-204 \pm 71$ & $-225 \pm 103$ & \\
\hline & 55339.206 & $-290 \pm 85$ & $-389 \pm 129$ & \\
\hline \multirow{2}{*}{ HD 328856} & 55336.370 & $-173 \pm 53$ & $-155 \pm 65$ & ND \\
\hline & 55339.223 & $-149 \pm 48$ & $-75 \pm 72$ & \\
\hline HD 152233 & 55339.289 & $-74 \pm 52$ & $-76 \pm 74$ & \\
\hline HD 152247 & 55339.261 & $34 \pm 52$ & $86 \pm 64$ & \\
\hline HD 152249 & 55339.275 & $-22 \pm 51$ & $-15 \pm 72$ & \\
\hline \multirow[t]{2}{*}{ HD 153426} & 55336.338 & $-27 \pm 53$ & $-10 \pm 62$ & \\
\hline & 55339.246 & $-171 \pm 55$ & $-275 \pm 70$ & ND \\
\hline HD 153919 & 55337.341 & $-213 \pm 68$ & $-119 \pm 95$ & ND \\
\hline HD 154368 & 55339.324 & $-74 \pm 38$ & $-77 \pm 63$ & \\
\hline HD 154643 & 55339.340 & $110 \pm 34$ & $121 \pm 52$ & ND \\
\hline HD 154811 & 55337.327 & $91 \pm 39$ & $59 \pm 64$ & \\
\hline HD 156154 & 55337.358 & $-118 \pm 38$ & $-167 \pm 54$ & ND \\
\hline HD 156212 & 55337.377 & $-104 \pm 42$ & $-51 \pm 63$ & \\
\hline HD 165319 & 55339.306 & $-44 \pm 48$ & $-38 \pm 74$ & \\
\hline HD 315033 & 55337.402 & $-41 \pm 41$ & $-36 \pm 52$ & \\
\hline HD 168075 & 55339.389 & $17 \pm 42$ & $3 \pm 65$ & \\
\hline \multirow[t]{2}{*}{ HD 168112} & 55336.418 & $-74 \pm 53$ & $-66 \pm 74$ & \\
\hline & 55339.362 & $112 \pm 62$ & $40 \pm 96$ & \\
\hline HD 169582 & 55339.415 & $-87 \pm 58$ & $-124 \pm 88$ & \\
\hline HD 171589 & 55339.445 & $-62 \pm 140$ & $-119 \pm 180$ & \\
\hline HD 175754 & 55337.426 & $82 \pm 67$ & $66 \pm 77$ & \\
\hline HD 187474 & 55339.433 & $-1249 \pm 22$ & $-1253 \pm 32$ & Ap star \\
\hline
\end{tabular}

Notes. All quoted errors are $1 \sigma$ uncertainties.

in the case of HD 148937 the confirmed detection is marked by CD.

Ten stars of our sample, CPD-28 2561, CPD-472963, HD 93843, HD 130298, HD 148937, HD 328856, HD 153426, HD 153919, HD 154643, and HD 156154 show evidence of a magnetic field.

Most importantly, the strongest magnetic fields are detected in both Of?p stars CPD-282561 and HD 148937. Walborn (1973) introduced the Of?p category for massive O stars displaying recurrent spectral variations in certain spectral lines, sharp emission or P Cygni profiles in He I and the Balmer lines, and strong C III emission lines around $4650 \AA$ A. Only five Galactic Of?p stars are presently known: HD 108, NGC 16242, CPD-28 2561, HD 148937, and HD 191612 (Walborn et al. 2010). Our observations of CPD-28 2561 enabled us to detect a magnetic field at the $3.1 \sigma$ level using the whole spectrum and at the $3.2 \sigma$ level using Balmer lines. The study of the radial velocity variation by Levato et al. (1988) found variability of a few emission lines with a probable period of 17 days. Walborn et al. (2010) report that CPD-28 2561 undergoes extreme spectral transformations very similar to those of HD 191612, on a timescale of weeks, inferred from the variable emission intensity of the C III $\lambda \lambda 4647-4650-4652$ triplet. The detection of a mean longitudinal magnetic field $\left\langle B_{z}\right\rangle=-254 \pm 81 \mathrm{G}$ in the Of?p star HD 148937 using FORS 1 at the VLT was previously reported by Hubrig et al. (2008). An extensive multiwavelength study of HD 148937 was carried out by Nazé et al. (2008), who detected the small-scale variations of He II 4686 and the Balmer lines with a period of seven days and an overabundance of nitrogen by a factor of four compared to the Sun. The periodicity of spectral variations in hydrogen and helium lines was reconfirmed using additional higher resolution spectroscopic material indicating the similarity to the other Of?p stars HD 108 and HD 191612 (Naze et al. 2010). Our spectropolarimetric observations of this star indicate that the magnetic field is variable, but owing to the low number of measurements it is not possible to verify the period deduced from spectroscopic observations. The magnetic field of this star was observed at $4.8 \sigma, 2.9 \sigma$, and $3.4 \sigma$ levels on three different nights using all absorption lines.

The remaining three Of?p stars are located in the northern hemisphere and cannot be reached with FORS 2 at the VLT. To study magnetic fields in HD 108 and HD 191612 we used polarimetric spectra obtained with the SOFIN spectrograph installed at the Nordic Optical Telescope (Hubrig et al. 2010). As a result, we detected a longitudinal magnetic field $\left\langle B_{z}\right\rangle=-168 \pm 35 \mathrm{G}$ in the Of?p star HD 108, which agrees with the longitudinal magnetic field measurement of the order of $-150 \mathrm{G}$ reported by Martins et al. (2010). For the star HD 191612 with a rotation period of 537.6 d (Howarth et al. 2007), we measured a longitudinal magnetic field $\left\langle B_{z}\right\rangle=450 \pm 153 \mathrm{G}$ at rotation phase 0.43 (Hubrig et al. 2010). The only previously published magnetic field measurement for this star showed a negative longitudinal magnetic field $\left\langle B_{z}\right\rangle=-220 \pm 38 \mathrm{G}$ at rotation phase 0.24 (Donati et al. 2006), indicating a change of polarity over $\sim 100$ days. No attempt has yet been made to measure the magnetic field of NGC 1624-2. Clearly these magnetic field measurements in Of?p stars imply that there is a tight relation between the observed properties of the Of?p star group and the presence of a magnetic field.

For the star CPD-47 2963, we achieved a 3.1 $\sigma$ detection using all absorption lines. According to Walborn et al. (2010), this star belongs to the Ofc category, which consists of normal spectra with C III $\lambda \lambda$ 4647-4650-4652 emission lines of comparable intensity to those of the Of defining lines N III $\lambda \lambda$ 4634-46404642. The authors indicate that the Ofc phenomenon occurs primarily in certain associations and young clusters. However, the available kinematic and photometric data do not indicate cluster or association membership for CPD-47 2963. The origin of the magnetic field in this star probably differs from those of other magnetic O-type stars, because non-thermal radio emission, which is frequently observed in binary systems with colliding winds, was detected by Benaglia et al. (2001). On the other hand, the membership of CPD-47 2963 in a binary or multiple system has not yet been investigated. The authors suggest that the non-thermal radiation from this star possibly originates from strong shocks in the wind itself and/or in the wind colliding region if the star has a massive early-type companion. Both optical and radio observations reveal the presence of a second source separated by $5^{\prime \prime}$.

According to Walborn et al. (2010), the star HD 93843, with a $3.7 \sigma$ detection achieved using all absorption lines, belongs to the Ofc category. Prinja et al. (1998) monitored the stellar 
wind of this star using IUE time series. They identified systematic changes in the absorption troughs of the Si IV and N v resonance lines with a repeatability of wind structures with a period of 7.1 days. The authors suggest that a magnetic field might help explain the cyclical wind perturbation. On the other hand, three other stars of the Ofc category included in our survey, HD 93204, HDE 303308, and HD 93403, do not have a detectable magnetic field at the $3 \sigma$ level.

The star HD 130298 with a longitudinal magnetic field observed at the $3.1 \sigma$ level using the Balmer lines, is known as an object with a bow shock. Noriega-Crespo et al. (1997) used ISSA/IRAS archival spectra to identify stars surrounded by extended infrared emission at $60 \mu \mathrm{m}$, which is a signature of wind bow shocks. The bow shocks are usually associated with runaway early-type stars with typical wind velocities of 500$3000 \mathrm{~km} \mathrm{~s}^{-1}$ and mass loss rates $\sim 10^{-5}-10^{-6} M_{\odot} \mathrm{yr}^{-1}$ (see e.g. Puls et al. 1996).

The two stars HD 328856 and HD 153426, which both have magnetic field detections, were observed on two different nights, namely the first and the fourth night of our observing run. For HD 328856, we obtained on these nights $3.3 \sigma$ and $3.1 \sigma$ level detections, respectively, using all absorption lines. On the basis of the photometric membership probability, this star is a member of the compact open cluster Hogg 22 in the Ara region at an age of 5 Myr and a distance of about 1300 pc (see Sect. 3). On the other hand, its proper motions indicate that HD 328856 is not fully co-moving with the other cluster members, deviating from the cluster mean proper motion by $\sim 2 \sigma$ (for more details of the membership probabilities, see Kharchenko et al. 2004). The observations of the star HD 153426 detected a mean longitudinal magnetic field at the $3.9 \sigma$ level using Balmer lines on the fourth night. The non-detection of the magnetic field for HD 153426 on the first observing night can probably be explained by the strong dependence of the longitudinal magnetic field on the rotational aspect. HD 153426 is a double-lined spectroscopic binary with unknown orbit parameters and was considered by de Wit et al. (2005) as a star in a newly detected cluster. Using the highest quality kinematic data on young open clusters presently available, Schilbach \& Röser (2008) suggested that HD 153426 was ejected from the cluster Hogg 22. Their back-tracing procedure indicates that the encounter time for HD 153426, i.e. the time when the star was ejected, is about 8.1 Myr, while the age of the cluster Hogg 22 is only 5 Myr.

The star HD 153919 was observed only once, revealing the presence of a mean longitudinal magnetic field at the $3.9 \sigma$ level, using all absorption lines. The study of Ankay et al. (2001) suggested that this star is a runaway X-ray binary, ejected from the OB association Sco OB1 about 2 Myr ago by the supernova of 4U1700-37's progenitor. They considered this star to be a companion of 4U1700-37, most likely a neutron star powered by wind accretion (e.g., Jones et al. 1973). Since 4U1700-37 is a candidate low-mass black hole (Brown et al. 1996), this system can be similar to the optical component (the O9.7 Iab supergiant) in the system Cyg X-1, for which a variable weak magnetic field was detected using a FORS 1 spectropolarimetric time series over the orbital period of 5.6 days (Karitskaya et al. 2010). Schilbach \& Röser (2008) identified the origin of this field star in the cluster NGC 6231 (the open cluster inside Sco OB1) at an age of about 6.5 Myr and their back-tracing procedure indicates that the star was ejected from the cluster 1.1 Myr ago.

The longitudinal magnetic field for the star HD 154643 was observed at the $3.2 \sigma$ level using all absorption lines. De Wit et al. (2005) characterise this star as a candidate runaway star associated with the young cluster Bochum 13. However,
Schilbach \& Röser (2008) identified the origin of this field star in the cluster ASCC 88, which has an an age of about $14.8 \mathrm{Myr}$, and proposed that HD 154643 was ejected 1.4 Myr ago.

The star HD 156154, for which we achieved a $3.1 \sigma$ detection using all absorption lines, seems to be the only star with a high cluster membership probability among the O-type stars with detected magnetic fields we have considered here (see Sect. 3). According to kinematic and photometric criteria, this star belongs to the open cluster Bochum 13 at an age of $12 \mathrm{Myr}$ and a distance of about $1 \mathrm{kpc}$.

\section{Discussion}

There has been much research of massive stars in recent years in order to properly model the effects of their rotation, stellar winds, and surface chemical composition. However, possible paths for the formation of magnetic O-type stars have not been yet analysed by modern evolution theories of single and binary stars. The number of massive stars with detected magnetic fields remains small, and the available data are insufficient to prove statistically whether magnetic fields in massive stars are ubiquitous or appear in specific stars with certain stellar parameters and a special environment. On the other hand, the observations of magnetic fields in massive stars accumulated over the past few years can be used to preliminarily constrain the conditions that enable the appearance of magnetic fields and provide the first trends about their occurrence and field strength distribution.

Since no longitudinal magnetic fields stronger than $300 \mathrm{G}$ have been detected in our study (apart from the rather strong field in the Of?p star CPD-282561), we have confirmed our previous conclusion (Hubrig et al. 2008) that large-scale, dipolelike, magnetic fields with polar field strengths higher than $1 \mathrm{kG}$ are not widespread among $\mathrm{O}$ type stars. Our study presents the results of a magnetic field survey of 36 massive stars. Among them, 19 stars have been found to be related to open clusters and associations of different ages. The data on the cluster membership of these probable cluster O-type stars are presented in Table 3. To increase the significance of our statistic assessment, we present in the same table the data for an additional six probable cluster O-type stars, which have been studied in recent years by Hubrig et al. (2008, 2009b, 2011a), marked for convenience by an asterisk. As database for the compilation of Table 3, we used the All-sky Compiled Catalogue of 2.5 million stars (ASCC-2.5, 3rd version) of Kharchenko \& Roeser (2009). We note that for the calculation of kinematic membership probability only proper motions were used. According to Dias et al. (2002; catalogue version 3.1 (24/11/2010)), one of the previously studied O-type stars, the star HD 152408 (Hubrig et al. 2008), is projected onto the cluster Collinder 316, which represents a large group of bright stars superposed on Trumpler 24 at the age $\log t=6.92$. We have not included this star in Table 3, as no membership criteria were discussed in this work. According to Mason et al. (1998) and Pourbaix et al. $(2004)^{2}$, among the stars presented in Table 3 , six stars, HD 47839, HD 135240, HD 152233, HD 153919, HD 154368 , and HD 167263, are members of spectroscopic binary systems, with orbital periods between 3.4 and 9247 days.

From the inspection of kinematic and photometric membership probabilities, and following the membership criteria described by Kharchenko et al. (2004), five stars in Table 3, CPD-58 2620, HD 93129B, HDE 303308, HD 105056, HD 152233, have a rather low cluster membership probability.

${ }^{2}$ http//sb9.astro.ulb.ac.be/mainform.cgi 
Table 3. Probable members in open clusters and associations.

\begin{tabular}{|c|c|c|c|c|c|c|c|c|}
\hline $\begin{array}{l}\text { Object } \\
\text { name }\end{array}$ & $\begin{array}{c}\text { ASCC } \\
\text { number }\end{array}$ & $\begin{array}{l}P_{\text {kin }} \\
{[\%]}\end{array}$ & $\begin{array}{c}P_{\text {phot }} \\
{[\%]}\end{array}$ & Cluster & $\begin{array}{l}\text { dist } \\
{[\mathrm{pc}]}\end{array}$ & $\begin{array}{l}\log t \\
{[\mathrm{yr}]}\end{array}$ & $\begin{array}{c}\left(\mu_{X}, \mu_{Y}, \sigma_{\mu}\right)^{\mathrm{star}} \\
{[\mathrm{mas} / \mathrm{yr}]}\end{array}$ & $\begin{array}{c}\left(\mu_{X}, \mu_{Y}, \sigma_{\mu}\right)^{\mathrm{cl}} \\
{[\mathrm{mas} / \mathrm{yr}]}\end{array}$ \\
\hline HD 47839* & 1021435 & 62 & 100 & NGC 2264 & 660 & 6.81 & $-3.84,-2.50,0.94$ & $-2.70,-3.50,0.25$ \\
\hline CPD-58 2620 & 2232461 & 44 & 1 & Trumpler 14 & 2753 & 6.67 & $-3.77,-2.20,2.37$ & $-3.91, \quad 3.65,0.60$ \\
\hline HDE 303311 & 2232607 & 91 & 27 & Trumpler 14 & 2753 & 6.67 & $-2.91,2.64,2.40$ & $3.65,0.60$ \\
\hline HD 93129B & 2232449 & 0 & 100 & Trumpler 14 & 2753 & 6.67 & $-9.80,10.86,1.96$ & $3.65,0.60$ \\
\hline HD 93204 & 2232588 & 75 & 0 & Trumpler 16 & 2842 & 6.90 & $-9.33, \quad 3.16,1.70$ & $4.02,0.42$ \\
\hline HDE 303308 & 2232708 & 19 & 0 & Trumpler 16 & 2842 & 6.90 & $-6.44, \quad 2.54,1.79$ & $-11.10, \quad 4.02,0.42$ \\
\hline HD 105056 & 2396832 & 12 & 100 & ASCC 69 & 1000 & 7.91 & $-4.56,-1.81,0.87$ & $7.91,-7.52,0.41$ \\
\hline HD 115455 & 2338543 & 90 & 72 & Stock 16 & 1640 & 6.78 & $-2.56, \quad 1.08,2.02$ & $-3.31,-0.03,0.66$ \\
\hline HD 120521 & 2256830 & 0 & 100 & Platais 10 & 246 & 8.20 & $-5.55, \quad 0.40,1.39$ & $-29.10,-10.73,0.40$ \\
\hline HD 123590 & 2345821 & 83 & 83 & ASCC 77 & 2200 & 6.99 & $-4.96,-2.45,0.99$ & $-4.59,-1.49,0.47$ \\
\hline HD 328856 & 2074567 & 22 & 100 & Hogg 22 & 1297 & 6.70 & $0.78,-1.37,1.26$ & $-0.84,-4.39,0.43$ \\
\hline HD $135240^{*}$ & 2351294 & 61 & 100 & ASCC 79 & 800 & 6.86 & $-3.00,-2.65,0.85$ & $-2.67,-4.10,0.44$ \\
\hline HD $135591^{*}$ & 2351425 & 67 & 100 & ASCC 79 & 800 & 6.86 & $-3.00,-2.65,0.85$ & $-2.67,-4.10,0.44$ \\
\hline HD 152233 & 1974789 & 13 & 100 & NGC 6231 & 1250 & 6.81 & $-4.11,-8.05,2.40$ & $-0.39,-1.99,0.46$ \\
\hline HD 152247 & 1974811 & 95 & 100 & NGC 6231 & 1250 & 6.81 & $-0.55,-1.14,1.91$ & $-0.39,-1.99,0.46$ \\
\hline HD 152249 & 1974812 & 84 & 100 & NGC 6231 & 1250 & 6.81 & $0.13,-0.81,1.36$ & $-0.39,-1.99,0.46$ \\
\hline HD 153919 & 1877055 & 0 & 100 & NGC 6281 & 494 & 8.51 & $1.42,4.84,1.04$ & $-2.96,-3.75,0.23$ \\
\hline HD 154368 & 1877523 & 88 & 100 & ASCC 88 & 1900 & 7.17 & $3.80,-2.08,1.05$ & $2.89,-2.00,0.32$ \\
\hline$H D 155806^{*}$ & 1783567 & 98 & 100 & Sco OB4 & 1100 & 6.82 & $0.33,-2.02,0.80$ & $0.46,-2.22,0.15$ \\
\hline HD 156154 & 1878846 & 82 & 100 & Bochum 13 & 1077 & 7.08 & $-0.88,-2.53,1.64$ & $-0.28,-1.20,0.30$ \\
\hline HD 164794* & 1593528 & 69 & 100 & NGC 6530 & 1322 & 6.67 & $1.92,-0.40,1.09$ & $2.01,-1.81,0.51$ \\
\hline HD 315033 & 1593655 & 97 & 100 & NGC 6530 & 1322 & 6.67 & $2.33,-2.51,2.09$ & $2.01,-1.81,0.51$ \\
\hline HD 167263* & 1595683 & 97 & 100 & Sgr OB7 & 1860 & 6.64 & $1.56,-1.53,1.19$ & $1.80,-1.20,0.26$ \\
\hline HD 168075 & 1407400 & 73 & 100 & NGC 6611 & 1719 & 6.72 & $2.81,-1.14,1.18$ & $1.60,-0.35,0.41$ \\
\hline HD 168112 & 1407419 & 73 & 19 & NGC 6604 & 1696 & 6.64 & $1.67,-2.20,1.69$ & $-0.36,-2.68,0.31$ \\
\hline HD 153426 & 1876676 & 0 & 0 & $\operatorname{Hogg} 22$ & 1297 & $70(6.91)$ & $-0.21,-0.09,1.38$ & $-0.84,-4.39,0.43$ \\
\hline HD 154643 & 1877779 & 0 & 0 & ASCC 88 & 1900 & $7.17(6.15)$ & $3.66,-1.71,1.55$ & $2.89,-2.00,0.32$ \\
\hline HD 169582 & 1323022 & 0 & 0 & NGC 6604 & 1696 & $6.64(6.74)$ & $-0.40,-1.63,1.14$ & $-0.36,-2.68,0.31$ \\
\hline HD 171589 & 1409170 & 0 & 0 & NGC 6618 & 1814 & $7.78(6.52)$ & $5.10, \quad 3.74,1.66$ & $3.04, \quad 0.33,0.92$ \\
\hline HD 175754 & 1503597 & 0 & 0 & ASCC 93 & 2500 & $7.72(7.01)$ & $1.49,2.15,1.01$ & $-2.18,-1.80,0.55$ \\
\hline
\end{tabular}

Notes. For each star, we give in the first two columns the object name and the corresponding catalogue number. The kinematic and photometric probabilities for cluster membership presented in Cols. 3 and 4 were calculated according to the procedures described by Kharchenko et al. (2004). The cluster names, the distances, and the ages are presented in the next three columns. The proper motions and their errors for stars and clusters are given in the last columns. All cluster data are taken from Kharchenko et al. (2005a,b). The five stars at the end of the table are currently not members of the indicated clusters, and were ejected from them $t_{\text {enc }}$ years ago (Schilbach \& Roeser 2008). The encounter times $t_{\text {enc }}$ of these stars are given in brackets. The three stars with magnetic fields that are most probably cluster members are presented in italics.

Two other stars, HD 120521 and HD 153919, are not kinematic members of the oldest clusters Platais 10 and NGC 6281, respectively, and should be regarded as field stars projected against the clusters by chance. Among the remaining stars, only in three stars, HD 155806, HD 156154, and HD 164794, have weak magnetic fields been detected (more details about the kinematic study of HD 155806 and HD 164794 can be found in Hubrig et al. 2011a). In Fig. 1, we display the positions of the stars listed in Table 3 in the colour-magnitude diagram. The absolute magnitudes $M_{\mathrm{V}}$ and intrinsic colours $(B-V)_{0}$ were calculated using cluster distances and $E(B-V)$ values presented by Kharchenko et al. (2005a,b). The theoretical isochrones were calculated by the Padova group (Girardi et al. 2002) and the values for the zero-age main sequence were retrieved from Schmidt-Kaler (1982). The absolute magnitudes and colours are presented in Table 4. The uncertainty in the distance modulus is assumed to be 0.2 . The positions of the magnetic stars with high cluster-membership probabilities, HD 156154, HD 155806, and HD 164794, do not represent any specific distribution that would indicate that their magnetic fields appear at a certain evolutionary age.

In Fig. 2, we present the age distribution of the most probable cluster members. While the age of HD 155806 and HD 164794 is similar to the bulk of the studied cluster O-type stars, the star
HD 156154 is somewhat older at an age of $\sim 12$ Myr. HD 155806 is classified as an Oe star, possibly representing the higher mass analogues of classical Be stars (e.g. Walborn 1973). Only six members have been proposed to belong to this group of stars (e.g. Negueruela et al. 2004). The star HD 164794 is a spectroscopic double-lined system with an orbital period of $2.4 \mathrm{yr}$, that is known to emit non-thermal radio-emission, probably associated with colliding winds (Nazé et al. 2010). No specific information can be found in the literature about the luminous supergiant HD 156154.

The available observations seem to indicate that a magnetic field is more frequently detected in field stars than in stars belonging to clusters or associations. It is generally accepted that the majority of massive stars form in star clusters and associations, and studies of kinematical properties of the massive star field population indicate that a major part of these stars can be traced back to their parent open clusters or associations (e.g. Schilbach \& Roeser 2008). Pflamm-Altenburg \& Kroupa (2010) discussed in their work whether massive stars can form in isolation in the galactic field. According to de Wit et al. (2005), only a few per cent of all O-type stars can be considered as having formed outside a cluster environment. Pflamm-Altenburg \& Kroupa considered the two-step-ejection process, which represents the combination of the dynamical and the supernova 


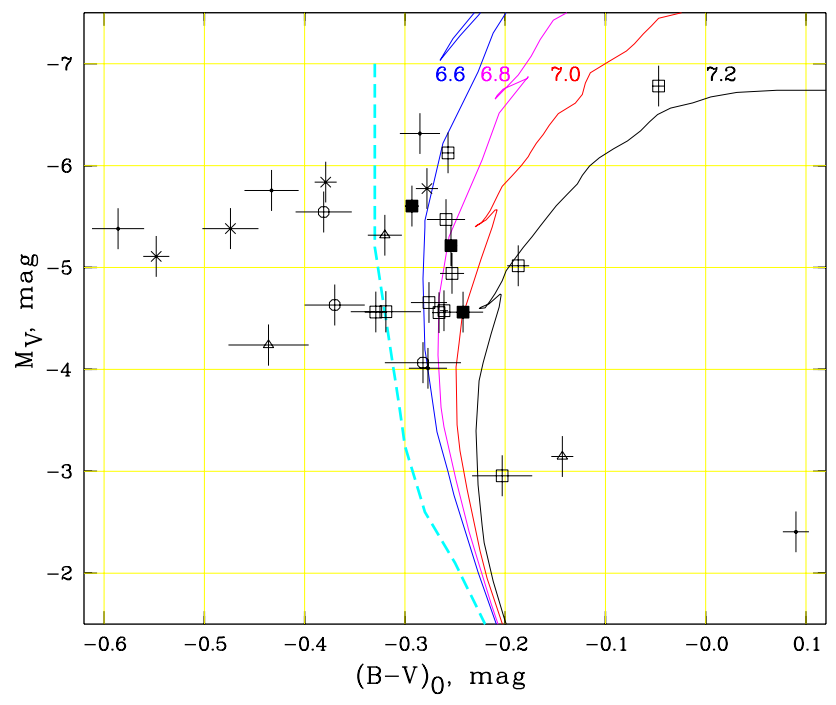

Fig. 1. The positions of the stars studied for cluster membership in the colour-magnitude diagram. Different symbols indicate stars with different membership probabilities: squares represent stars with cluster membership probability higher than $60 \%$, circles stars with a probability between $14 \%$ and $60 \%$, and triangles membership probability between $1 \%$ and $14 \%$. Non-members and runaway stars are marked by dots and crosses, respectively. One runaway star, HD 171589, does not appear in this figure, as its colour and magnitude do not fit the presented parameter space (see Table 4). The three stars with magnetic fields, HD 155806, HD 156154, and HD 164794, with high cluster membership probabilities are denoted by filled squares. Isochrones for $\log t=6.6,6.8,7.0$, and 7.2 are presented by solid lines and the zero-age main sequence is shown as a dashed line.

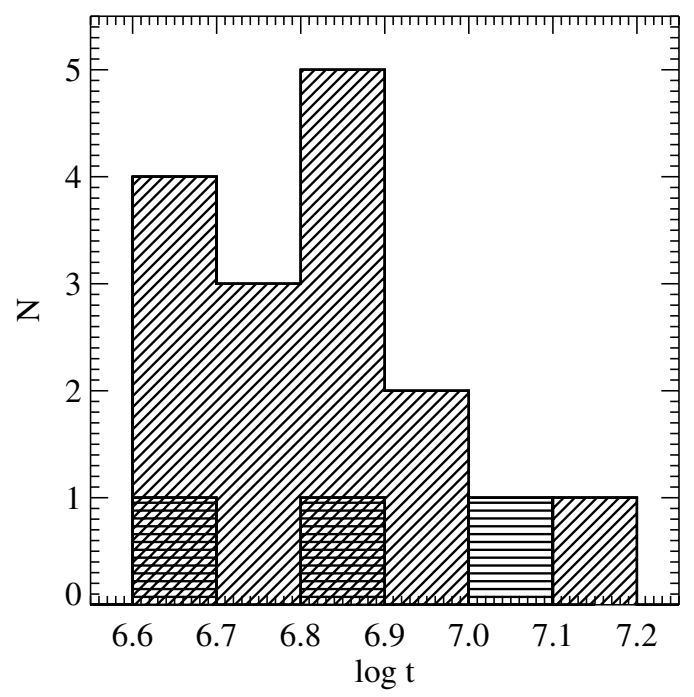

Fig. 2. The age distribution of studied probable cluster members. The three stars with magnetic fields, HD 155806, HD 156154, and HD 164794, are denoted by horizontal lines.

ejection scenario with the result that massive field stars produced via this ejection process in the vast majority of cases cannot be traced back to their parent star clusters. These stars might be mistakenly considered as massive stars formed in isolation. While this cannot be proven, the observed numbers of field $\mathrm{O}$ stars is consistent with this idea.

For the newly detected magnetic O-type stars, HD 153426 , HD 153919, and HD 154643, Schilbach \& Roeser (2008) suggested that the three stars were ejected from the clusters
Table 4. Absolute magnitudes and intrinsic colours of stars studied for the cluster membership.

\begin{tabular}{lcc}
\hline \hline Object name & $M_{\mathrm{V}}$ & $(B-V)_{0}$ \\
\hline HD 47839 & -4.557 & $-0.266 \pm 0.007$ \\
CPD-58 2620 & -4.239 & $-0.436 \pm 0.040$ \\
HDE 303311 & -4.632 & $-0.370 \pm 0.030$ \\
HD 93129B & -6.315 & $-0.285 \pm 0.020$ \\
HD 93204 & -5.381 & $-0.586 \pm 0.026$ \\
HDE 303308 & -5.756 & $-0.433 \pm 0.027$ \\
HD 105056 & -3.145 & $-0.143 \pm 0.011$ \\
HD 115455 & -4.563 & $-0.329 \pm 0.011$ \\
HD 120521 & -4.011 & $-0.277 \pm 0.019$ \\
HD 123590 & -5.017 & $-0.187 \pm 0.011$ \\
HD 328856 & -4.066 & $-0.282 \pm 0.038$ \\
HD 135240 & -4.942 & $-0.253 \pm 0.012$ \\
HD 135591 & -4.576 & $-0.261 \pm 0.004$ \\
HD 152233 & -5.317 & $-0.320 \pm 0.017$ \\
HD 152247 & -4.656 & $-0.276 \pm 0.018$ \\
HD 152249 & -5.471 & $-0.259 \pm 0.019$ \\
HD 153919 & -2.406 & $0.090 \pm 0.013$ \\
HD 154368 & -6.781 & $-0.047 \pm 0.006$ \\
HD 155806 & -5.214 & $-0.254 \pm 0.006$ \\
HD 156154 & -4.562 & $-0.242 \pm 0.020$ \\
HD 164794 & -5.603 & $-0.293 \pm 0.007$ \\
HD 315033 & -2.956 & $-0.203 \pm 0.030$ \\
HD 167263 & -6.125 & $-0.257 \pm 0.006$ \\
HD 168075 & -4.566 & $-0.319 \pm 0.035$ \\
HD 168112 & -5.545 & $-0.381 \pm 0.028$ \\
\hline HD 153426 & -5.109 & $-0.548 \pm 0.013$ \\
HD 154643 & -5.775 & $-0.278 \pm 0.011$ \\
HD 169582 & -5.382 & $-0.474 \pm 0.028$ \\
HD 171589 & -8.036 & $-1.340 \pm 0.021$ \\
HD 175754 & -5.838 & $-0.379 \pm 0.011$ \\
\hline & &
\end{tabular}

Hogg 22, NGC 6231, and ASCC 88, respectively. On the other hand, none of the four magnetic Of?p stars is known to belong to a cluster or an association. The study of the evolutionary state of HD 108 and HD 191612 indicates that both stars have evolved significantly (Martins et al. 2010). Our kinematic study of the Of?p star HD 148937 has shown that it possesses a space velocity of $32 \mathrm{~km} \mathrm{~s}^{-1}$ with respect to the Galactic open cluster system, with the velocity component $U=-26$ being directed in the opposite direction from the Galactic center and the velocity component $W=-13$ directed from the Galactic plane (Hubrig et al. 2011a). These rather large velocities indicate that this star can be considered as a candidate runaway star.

It is striking that most previously detected magnetic O-type stars are candidate runaway stars (Hubrig et al. 2011a,c). In the sample of O-stars with magnetic fields detected in this work, four other stars, HD 130298, HD 153426, HD 153919, and HD 154643, are also mentioned in the literature as candidate runaway stars. In the past, two mechanisms have been discussed to explain the existence of runaway stars: in one scenario, close multi-body interactions in a dense cluster environment cause one or more stars to be scattered out of the region (e.g. Leonard \& Duncan 1990). For this mechanism, runaways are ejected in dynamical three- or four-body interactions. An alternative mechanism involves a supernova explosion within a close binary, ejecting the secondary according to the conservation of momentum (Zwicky 1957; Blaauw 1961). However, none of these scenarios consider the possibility that a massive star can acquire a magnetic field during the ejection process. These findings clearly provide a strong motivation to carry out a kinematic study of all stars previously surveyed for magnetic fields to search for a 
correlation between the kinematic status and the presence of a magnetic field.

On the basis of these still very limited magnetic surveys of massive stars, we are unable to conclude whether O-type stars are magnetic in certain evolutionary states and a specific environment. Open star clusters and associations are very useful laboratories for testing star formation and stellar evolution. The ages of our subsample of three stars with magnetic fields do not contradict the idea that it is drawn from the general distribution of cluster ages. We have to keep in mind though that we have a very small number statistics. It appears that our observations are consistent with the assumption that the presence of a magnetic field can be expected in stars of different classification categories. Although a few hot magnetic stars might be peculiar on the basis of their spectral morphology, prior to their field detection (e.g. Of?p; Walborn 2006), the presence of a magnetic field can also be expected in stars of other classifications. Future magnetic field measurements are urgently needed to constrain the conditions controlling the presence of magnetic fields in hot stars, and the implications of these fields for their physical parameters and evolution.

Acknowledgements. A.E.P. acknowledges support of the RFBR grant 10-0291338 .

\section{References}

Alves, F. O., Franco, G. A. P., \& Girart, J. M. 2008, A\&A, 486, L13 Ankay, A., Kaper, L., de Bruijne, J. H. J., et al. 2001, A\&A, 370, 170 Benaglia, P., Cappa, C. E., \& Koribalski, B. S. 2001, A\&A, 372, 952 Blaauw, A. 1961, Bull. Astron. Inst. Netherlands, 15, 265 Bonanno, A., \& Urpin, V. 2008, MNRAS, 388, 1679

Brown, G. E., Weingartner, J. C., \& Wijers, R. A. M. J. 1996, ApJ, 463, 297 de Wit, W. J., Testi, L., Palla, F., \& Zinnecker, H. 2005, A\&A, 437, 247 Dias, W. S., Alessi, B. S., Moitinho, A., \& Lépine, J. R. D. 2002, A\&A, 389, 871 Donati, J.-F., Howarth, I. D., Bouret, J.-C., et al. 2006, MNRAS, 365, L6 Girardi, L., Bertelli, G., Bressan, A., et al. 2002, A\&A, 391, 195 Gómez, G. C., \& Cox, D. P. 2004, ApJ, 615, 744

Howarth, I. D., Walborn, N. R., Lennon, D. J., et al. 2007, MNRAS, 381, 433 Hubrig, S., Kurtz, D. W., Bagnulo, S., et al. 2004a, A\&A, 415, 661
Hubrig, S., Szeifert, T., Schöller, M., et al. 2004b, A\&A, 415, 685 Hubrig, S., Briquet, M., Schöller, M., et al. 2006, MNRAS, 369, L61 Hubrig, S., Schöller, M., Schnerr, R. S., et al. 2008, A\&A, 490, 793 Hubrig, S., Schöller, M., Savanov, I., et al. 2009a, AN, 330, 708 Hubrig, S., Stelzer, B., Schöller, M., et al. 2009b, A\&A, 502, 283 Hubrig, S., Ilyin, I., \& Schöller, M. 2010, AN, 331, 781

Hubrig, S., Kharchenko, N. V., \& Schöller, M. 2011a, AN, 332, 65 Hubrig, S., Schöller, M., Ilyin, I., et al. 2011b, ApJ, 726, L5

Hubrig, S., Oskinova, L. M., \& Schöller, M. 2011c, AN, 332, 147

Jones, C., Forman, W., Tananbaum, H., et al. 1973, ApJ, 181, L43

Karitskaya, E. A., Bochkarev, N. G., Hubrig, S., et al. 2010, IBVS, 5950, 1

Kharchenko, N. V., \& Roeser, S. 2009, All-sky Compiled Catalogue of 2.5 million stars

Kharchenko, N. V., Piskunov, A. E., Röser, S., et al. 2004, AN, 325, 740

Kharchenko, N. V., Piskunov, A. E., Röser, S., Schilbach, E., \& Scholz, R.-D. 2005a, A\&A, 438, 1163

Kharchenko, N. V., Piskunov, A. E., Röser, S., Schilbach, E., \& Scholz, R.-D. 2005b, A\&A, 440, 403

Landstreet, J. D., \& Mathys, G. 2000, A\&A, 359, 213

Leonard, P. J. T., \& Duncan, M. J. 1990, AJ, 99, 608

Levato, H., Morrell, N., Garcia, B., \& Malaroda, S. 1988, ApJS, 68, 319

Maíz-Apellániz, J., Walborn, N. R., Galué, H. Á., \& Wei, L. H. 2004, ApJS, 151, 103

Martins, F., Donati, J.-F., Marcolino, W. L. F., et al. 2010, MNRAS, 407, 1423

Mason, B. D., Gies, D. R., Hartkopf, W. I., et al. 1998, AJ, 115, 821

Nazé, Y., Walborn, N. R., Rauw, G., et al. 2008, AJ, 135, 1946

Nazé, Y., ud-Doula, A., Spano, M., et al. 2010, A\&A, 520, A59

Negueruela, I., Steele, I. A., \& Bernabeu, G. 2004, AN, 325, 749

Noriega-Crespo, A., van Buren, D., \& Dgani, R. 1997, AJ, 113, 780

Pflamm-Altenburg, J., \& Kroupa, P. 2010, MNRAS, 404, 1564

Pourbaix, D., Tokovinin, A. A., Batten, A. H., et al. 2004, A\&A, 424, 727

Prinja, R. K., Massa, D., Howarth, I. D., \& Fullerton, A. W. 1998, MNRAS, 301, 926

Puls, J., Kudritzki, R.-P., Herrero, A., et al. 1996, A\&A, 305, 171

Schilbach, E., \& Röser, S. 2008, A\&A, 489, 105

Schmidt-Kaler, Th. 1982, in Landolt-Börnstein Numerical Data and Functional Relationships in Science and Technology, New Series, Group IV, ed. K. Schafer, \& H. H. Voigt (Berlin-Heidelberg, New York: Springer-Verlag Press)

Walborn, N. R. 1973, AJ, 78, 1067

Walborn, N. R. 2006, in The Ultraviolet Universe: Stars from Birth to Death, 26th meeting of the IAU, Joint Discussion 4, 16-17 August, Prague, Czech Republic, JD04, \#19

Walborn, N. R., Sota, A., Maíz Apellániz, J., et al. 2010, ApJ, 711, L143

Zwicky, F. 1957, Morphological astronomy (Berlin: Springer) 